\title{
REMARKS ON COMPUTING IRREDUCIBLE CHARACTERS
}

\author{
G. LUSZTIG
}

\section{INTRODUCTION}

0.1 . Let $G$ be a connected reductive algebraic group defined over a finite field $F_{q}$ and let $G\left(F_{q}\right)$ be the finite group of all $F_{q}$-rational points of $G$.

We would like to present here a strategy for computing the character table of $G\left(F_{q}\right)$, under the assumption that $p$, the characteristic of $F_{q}$, is sufficiently large.

We can assume that $G$ has a simply connected derived group. Indeed, in the general case, we can find a connected reductive group $\widetilde{G}$ with simply connected derived group, and a surjective homomorphism $f: \widetilde{G} \rightarrow G$ whose kernel is a central torus such that $\widetilde{G}$ and $f$ are defined over $F_{q}$; then the character table of $G\left(F_{q}\right)$ can be easily recovered from that of $\widetilde{G}\left(F_{q}\right)$.

Let $l$ be a prime number different from $p$. We consider the vector space $\mathscr{A}$ of class functions $G\left(F_{q}\right) \rightarrow \bar{Q}_{l}$.

There are two important bases for $\mathscr{A}$. The first one, denoted $\mathbf{B}_{0}$, is well understood; it is given by the characteristic functions $f_{x}: G\left(F_{q}\right) \rightarrow \bar{Q}_{l}$ of the various $G\left(F_{q}\right)$-conjugacy classes $x$ (here, $f_{x}$ takes the value 1 on $x$ and the value 0 on the complement of $x$ ).

The second one, denoted $\mathbf{A}_{0}$, consists of the irreducible characters of $G\left(F_{q}\right)$. These have been classified in [L3, L7] in a way which is almost totally unrelated with the basis $\mathbf{B}_{0}$.

To find the character table of $G\left(F_{q}\right)$, we must express the basis $\mathbf{B}_{0}$ explicitly in terms of the basis $\mathbf{A}_{0}$.

\section{2 . We recall a well-known definition.}

Let $C$ be a locally closed, smooth, irreducible subvariety of $G$ which is stable under conjugation by $G$ and is defined over $F_{q}$; let $\mathscr{E}$ be an irreducible $G$-equivariant $\bar{Q}_{l}$-local system on $C$ such that the inverse image $F^{*} \mathscr{E}$ of $\mathscr{E}$ under the Frobenius map $F: G \rightarrow G$ is isomorphic to $\mathscr{E}$. Choose an isomorphism of local systems $\pi: \mathscr{E} \cong F^{*} \mathscr{E}$. Let $\mathscr{E}^{\sharp}$ be the intersection cohomology complex of the closure $\bar{C}$ of $C$ in $G$, with coefficients in $\mathscr{E}$. Then $\pi$ extends naturally to an isomorphism $\mathscr{E}^{\sharp} \cong F^{*} \mathscr{E}^{\sharp}$; taking the alternating sum of traces of the automorphisms induced on the stalks of the cohomology sheaves of $\mathscr{E}^{\sharp}$

Received by the editors November 6, 1991.

1991 Mathematics Subject Classification. Primary 20 G99.

Supported in part by National Science Foundation grant DMS 8702842. 
at rational points of $\bar{C}$, we obtain a function $\bar{C}\left(F_{q}\right) \rightarrow \bar{Q}_{l}$; extending this by 0 on $G\left(F_{q}\right)-\bar{C}\left(F_{q}\right)$, we obtain a class function $\chi_{C, \mathscr{E}, \pi}: G\left(F_{q}\right) \rightarrow \bar{Q}_{l}$.

Let $q_{C}=q^{\operatorname{codim} C / 2}$. (For any integer $n$ we denote $q^{n / 2}=\left(q^{1 / 2}\right)^{n}$ where $q^{1 / 2}$ is a fixed square root of 1 in $\bar{Q}_{l}$.)

0.3. Let us take all $(C, \mathscr{E})$ as above, where $C$ is a (variable) conjugacy class and $\mathscr{E}$ is given up to isomorphism.

For each such $(C, \mathscr{E})$ we choose $\pi$ in 0.2 such that

(a) the induced automorphism $\pi_{c}$ of the stalk $\mathscr{E}_{c}$ at $c$ has finite order for any $c \in C\left(F_{q}\right)$.

The resulting set of class functions $q_{C} \chi_{C, \mathscr{E}, \pi}$ is known to be a basis of $\mathscr{A}$. We denote this basis by $\mathbf{B}_{1}$.

The transition matrix from the basis $\mathbf{B}_{1}$ to the basis $\mathbf{B}_{0}$ is explicitly computable, from the results in [L5, §24] (which complete earlier results of [BS, S1]).

Thus the task of computing the character table of $G\left(F_{q}\right)$ is equivalent to the following problem:

(b) find explicitly the transition matrix from the basis $\mathbf{B}_{1}$ to the basis $\mathbf{A}_{1}$.

0.4. We want to state two other problems closely related to the problem $0.3(\mathrm{~b})$.

Let $\mathscr{Z}$ be the centre of $G$ and let $\mathscr{Z}^{0}$ be its identity component.

Let $(C, \mathscr{E})$ be as in 0.2 ; we assume that $C$ is a single $G \times \mathscr{Z}^{0}$-orbit on $G$ (the action of $G$ is by conjugation, while that of $\mathscr{Z}^{0}$ is by translation); we assume also that $\mathscr{E}$ is "cuspidal" in the sense of [L5] (this implies in particular that $\mathscr{E}^{\sharp}$ is zero on $\bar{C}-C$ ). Choose $\pi$ as in 0.2 so that the condition $0.3(\mathrm{a})$ is satisfied.

The resulting class functions $q_{C} \chi_{C, \mathscr{E}, \pi}$ are known to be linearly independent. They form a subset $\mathbf{B}_{2}$ of $\mathscr{A}$. (When $G$ is semisimple, $\mathbf{B}_{2}$ is a subset of $\mathbf{B}_{1}$.)

We state the following problems:

(a) express the functions of $\mathbf{B}_{2}$ as explicit linear combinations of functions in $\mathbf{A}_{0}$;

(b) find the explicit decomposition in irreducible characters of $R_{L}^{G}$ applied to a given irreducible character of $L\left(F_{q}\right)$.

$\left(R_{L}^{G}\right.$ is the process of "twisted induction" (see [DL,L1]) which attaches a virtual representation of $G\left(F_{q}\right)$ to any virtual representation of $L\left(F_{q}\right)$, where $L$ is a Levi subgroup (defined over $F_{q}$ ) of parabolic subgroups (not necessarily defined over $F_{q}$ ); this is a generalization of the classical induction of characters, due to Frobenius.)

According to $[L 8,9.5]$, any function in the basis $\mathbf{B}_{1}$ can be expressed explicitly in terms of "twisted inductions" $R_{L}^{G}$ applied to functions in the sets $\mathbf{B}_{2}$ attached to various $L$ as above.

This means that problem $0.3(\mathrm{~b})$ (and hence the problem of computing the character table of $G\left(F_{q}\right)$ ) is reduced to problems (a) and (b).

0.5. In the sequel we shall assume that problem $0.4(\mathrm{~b})$ is solved. In the case 
where $L$ is a maximal torus, problem $0.4(\mathrm{~b})$ was solved in [L3, L7] at the same time as giving the classification of irreducible characters of $G\left(F_{q}\right)$; the coefficients in the the decomposition have been expressed there in terms of certain nonabelian Fourier transforms over some small finite groups. The same problem for general $L$ has been solved in [A, S2] assuming connected centre; this assumption should not be necessary.

0.6. We shall assume that $G$ is simply connected, almost simple. (The general case cannot be formally reduced to this case, but it can be treated by similar methods.)

It will be convenient to further modify our (partial) basis $\mathbf{B}_{2}$. The group $\mathscr{Z}\left(F_{q}\right)$ acts naturally on the vector space $\mathscr{A}$ by $\left(t_{z} f\right)(g)=f(z g)$ for all $z \in \mathscr{Z}\left(F_{q}\right), f \in \mathscr{A}, g \in G\left(F_{q}\right)$. One can normalize the elements of $\mathbf{B}_{2}$ so that $\mathscr{Z}\left(F_{q}\right)$ permutes the elements of $\mathbf{B}_{2}$ among themselves.

Let us pick an element $b \in \mathbf{B}_{2}$ in each $\mathscr{Z}\left(F_{q}\right)$-orbit and let us denote by $I_{b}$ its isotropy group in $\mathscr{Z}\left(F_{q}\right)$; for each character $\zeta: \mathscr{Z}\left(F_{q}\right) \rightarrow \bar{Q}_{l}^{*}$ which is trivial on $I_{b}$, we set $b(\zeta)=\left|I_{b}\right|^{-1} \sum_{z \in \mathscr{Z}\left(F_{q}\right)} \zeta\left(z^{-1}\right) t_{z} b$. Let $\mathbf{B}_{3}$ be the set of functions $b(\zeta)$ thus obtained. Clearly, $\mathbf{B}_{3}$ is a linearly independent set; it spans the same subspace of $\mathscr{A}$ as $\mathbf{B}_{2}$. Since the relation between $\mathbf{B}_{2}$ and $\mathbf{B}_{3}$ is quite explicit, we see that problem $0.4(\mathrm{a})$ is equivalent to the following problem:

(a) express the functions of $\mathbf{B}_{3}$ as explicit linear combinations of functions in $\mathbf{A}_{0}$.

We now see that our problem is essentially reduced to solving problem (a).

Our main result (Theorem 0.8 below) gives something close to a solution of problem (a) (the roots of 1 in its statement remain to be described). To state the theorem we need some further notation.

0.7. Let $\mathbf{A}_{1}$ be the orthonormal basis of $\mathscr{A}$ consisting of "almost characters". (This is defined for groups with connected centre in [L3]; the same definition can be given in general, in the framework of [L7].) By definition, the transition matrix from $\mathbf{A}_{1}$ to $\mathbf{A}_{0}$ is explicitly known: it involves the entries of certain nonabelian Fourier transforms.)

Let $\mathscr{A}^{0}$ be the space of all functions in $\mathscr{A}$ which are orthogonal to $R_{L}^{G}(f)$ for any proper $L$, defined over $F_{q}$ (as in 0.4$)$ and any virtual character $f$ on $L\left(F_{q}\right)$.

By the solution of problem $0.4(\mathrm{~b})$, the set $\mathbf{A}_{2}=\mathbf{A}_{1} \cap \mathscr{A}^{0}$ is a basis of $\mathscr{A}^{0}$; the functions in $\mathbf{A}_{2}$ are explicitly known as linear combinations of irreducible characters.

We will modify the functions in $\mathbf{A}_{2}$ by a procedure similar to the one in 0.6.

It is clear that the action of $\mathscr{Z}\left(F_{q}\right)$ on $\mathscr{A}$ (see 0.6 ) is such that $t_{z}$ maps each element of $\mathbf{A}_{0}$ to a (known) multiple of itself (by Schur's lemma). One verifies that this action is such that it permutes the elements of $\mathbf{A}_{1}$ among themselves and the elements of $\mathbf{A}_{2}$ among themselves; these permutations can be described explicitly.

Let us again pick an element $\beta \in \mathbf{A}_{2}$ in each $\mathscr{Z}\left(F_{q}\right)$-orbit on $\mathbf{A}_{2}$ and let us denote by $I_{\beta}$ its isotropy group in $\mathscr{Z}\left(F_{q}\right)$; for each character $\zeta: \mathscr{Z}\left(F_{q}\right) \rightarrow \bar{Q}_{l}^{*}$, 
we set $\beta(\zeta)=\left|I_{\beta}\right|^{-1} \sum_{z \in \mathscr{Z}\left(F_{q}\right)} \zeta\left(z^{-1}\right) t_{z} \beta$. Let $\mathbf{A}_{3}$ be the set of functions $\beta(\zeta)$ thus obtained.

Clearly, $\mathbf{A}_{3}$ is a linearly independent set; it spans the same subspace of $\mathscr{A}$ as $\mathbf{A}_{2}$ and the elements of this set are explicit linear combinations of irreducible characters.

We can now state:

Theorem 0.8. Given $f \in \mathscr{A}$, we have $f \in \mathbf{A}_{3}$ if and only if $\mu f \in \mathbf{B}_{3}$ for some root of unity $\mu$.

The correspondence between the labellings of the elements of these two sets of functions can also be found explicitly.

It follows that the pattern of decomposition of the functions in $\mathbf{B}_{3}$, as linear combinations of irreducible characters, is the same as that of the corresponding functions in $\mathbf{A}_{3}$; hence it is explicitly known, as required by problem $0.6(\mathrm{a})$.

0.9 . We note that a result like Theorem 0.8 has been previously known in a number of cases. The statement of Theorem 0.8 is part of a wider conjecture, involving character sheaves, which is formulated in [L3,L5].

In [L6] it was shown that the functions of $\mathbf{B}_{2}$ which have unipotent support and come from the adjoint group are contained in $\mathbf{A}_{2}$ (under the assumption that $G$ is split, and with some mild restrictions on $q$ ); this was proved independently, at about the same, by Kawanaka [K1], for groups of type $E_{8}$ and $F_{4}$, by quite different methods. Later, Kawanaka [K2] announced that he can prove a result which amounts to the equality $\mathbf{B}_{2}=\mathbf{A}_{2}$ for groups of type $E_{8}$ and $F_{4}$, using generalized Gelfand-Graev representations. After writing [L9], I could reconstruct (what I imagine that must have been) Kawanaka's proof; I could prove Theorem 0.8 with the same methods whenever $G$ has rank $\leq 8$. Thus Theorem 0.8 for rank $\leq 8$ should be attributed to Kawanaka. We will prove it for groups of rank $>8$, when different techniques are needed.

\section{CUSPidal Character SHEaves}

1.1. Let $G$ be a connected, semisimple algebraic group over $k$, an algebraic closure of the finite field with $p$ elements. We assume that $p$ is sufficiently large, so that the results of [L9] are applicable. We fix a prime number $l$ different from $p$.

We recall the definition of cuspidal character sheaves on $G$ (see [L5]) in a form convenient for our present purpose.

Let $J$ be the set of all pairs $(C, \mathscr{E})$ where $C$ is a conjugacy class in $G$ and $\mathscr{E}$ is an irreducible $\bar{Q}_{l}$-local system on $C$, equivariant for the conjugation action of $G$ on $C$ and given up to isomorphism. For $(C, \mathscr{E}) \in J$, we denote by $P_{C, \mathscr{E}}$ the irreducible perverse sheaf on $G$ whose support is the closure $\bar{C}$ of $C$ and whose restriction to $C$ is $\mathscr{E}$, up to shift.

We consider $J$ as set of vertices of an oriented graph: we have an edge from $(C, \mathscr{E})$ to $\left(C^{\prime}, \mathscr{E}^{\prime}\right)$ precisely when $C^{\prime} \subset \bar{C}-C$ and some cohomology sheaf of $P_{C, \mathscr{E}}$ restricted to $C^{\prime}$ contains $\mathscr{E}^{\prime}$ as a direct summand.

A pair $(C, \mathscr{E}) \in J$ is said to be cuspidal if it is an isolated vertex of the graph above, i.e., it is not joined with any other element of $J$. This is equivalent to 
the condition that $P_{C, \mathscr{E}}$ is a character sheaf of $G$ and also to the condition that $P_{C, \mathscr{E}}$ is a cuspidal character sheaf of $G$.

Let $J_{0}$ be the subset of $J$ consisting of cuspidal pairs. Then $(C, \mathscr{E}) \mapsto P_{C, \mathscr{E}}$ is a 1-1 correspondence between $J_{0}$ and the set of cuspidal character sheaves of $G$.

1.2. Let $G^{*}$ be the Langlands dual of $G$; this is another connected semisimple algebraic group over $k$, well defined by $G$ up to isomorphism. One of the main results of [L5] is a parametrization of the set of character sheaves of $G$ in terms of $G^{*}$. In particular, we have a canonical surjective map $\Psi$ from the set of character sheaves of $G$ to the set of special conjugacy classes of $G^{*}$. Recall that an element $g \in G^{*}$ or its conjugacy class is said to be special if the unipotent part of $g$ is a special unipotent element [L2] of the centralizer in $G^{*}$ of the semisimple part of $g$.

(In [L5] we have associated with each character sheaf on $G$ a local system on a maximal torus of $G$ and a two-sided cell in its stabilizer in the Weyl group; this can be interpreted as a semisimple element of $G^{*}$ and a special unipotent element element in its centralizer, hence as a special element of $G^{*}$, well defined up to conjugacy.)

By restricting $\Psi$ to the set of cuspidal character sheaves, identified as above with $J_{0}$, we obtain a map $\Psi_{0}$ from $J_{0}$ to the set of special conjugacy classes in $G^{*}$; let $S_{0}$ be its image.

Note that $J_{0}$ is a finite set, by [L4]; hence $S_{0}$ is a finite set.

1.3. There is a canonical surjective map $\Phi$ from the set of special conjugacy classes in $G^{*}$ to the set of unipotent classes in $G$; see $[\mathrm{L} 3,13.3 ; \mathrm{L} 9,10.5]$. This map preserves the codimension of a class.

By restricting $\Phi$ to $S_{0}$ we obtain a map from $S_{0}$ to the set of unipotent classes in $G$. The image of this map is denoted $U_{0}$. Thus $\Phi$ restricts to a surjective map $\Phi_{0}: S_{0} \rightarrow U_{0}$.

In the remainder of this section, we assume that $G$ is almost simple, simply connected. In 1.4-1.12 we will describe the set $S_{0}$ in each case; this description can be extracted from [L5].

We shall also describe in each case the set $U_{0}$ and the map $\Phi_{0}: S_{0} \rightarrow U_{0}$; this description is obtained from the definition of $\Phi_{0}$.

We will specify semisimple classes by indicating the type of the centralizer of an element in the class; this will be unambiguous in each case. We generally denote by $u$ an element of a class in $U_{0}$ and by $\sigma v=v \sigma$ an element of a class in $S_{0}$, where $\sigma \in G^{*}$ is semisimple and $v$ is unipotent in the centalizer $Z(\sigma)$ of $\sigma$ in $G^{*}$. We will specify unipotent classes in a reductive group by specifying their type in each simple factor (unipotent classes are not sensitive to isogeny); for exceptional factors we specify them by their Dynkin diagram while for factors isogenous to a classical group $S L_{n}, S O_{n}, S p_{2 n}$ we specify them by giving the types of Jordan blocks they have in the standard representation of that classical group. $J_{t}$ denotes a Jordan block of size $t$.

We will denote $\diamond=\{0,1,4,9, \ldots\}, \triangle=\{0,1,3,6,10,15, \ldots\}$.

1.4. $\left(G=S L_{n}(k), G^{*}=P G L_{n}(k), n \geq 2\right.$.) 
$S_{0}$ has a single element: the image in $P G L_{n}(k)$ of the class of a diagonal matrix in $G L_{n}(k)$ with distinct entries given by the various elements of $k$ whose $n$th power is 1 . (We assume $p>n$.)

$U_{0}$ has a single element: the regular unipotent class. The map $\Phi_{0}$ is the obvious one.

\section{5. $w\left(G=G^{*}=E_{8}(k)\right.$.)}

Both $S_{0}$ and $U_{0}$ consist of a single unipotent class, namely that of type 0002000 . The map $\Phi_{0}$ is the obvious one.

1.6. $\left(G=E_{7}(k)^{\mathrm{sc}}, G^{*}=E_{7}(k)^{\text {ad }}\right.$.)

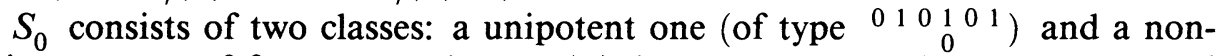
unipotent one of form $\sigma v$, where $Z(\sigma)$ has two connected components, and its identity component has one-dimensional centre and an adjoint group of type $E_{6}$; the element $v$ of this centralizer has type $\begin{gathered}0 \\ 0\end{gathered} 200$.

$U_{0}$ consists of two unipotent classes. One is of type 010101 (it is the image under $\Phi_{0}$ of the unipotent class in $S_{0}$ ):

The other one is of type 200200 (it is the image under $\Phi_{0}$ of the nonunipotent class in $S_{0}$ ).

1.7. $\left(G=E_{6}(k)^{\mathrm{sc}}, G^{*}=E_{6}(k)^{\text {ad }}\right.$.)

$S_{0}$ consists of two classes: a unipotent one (of type $\begin{array}{ccc}0 & 0200 & 0\end{array}$ ) and a nonunipotent one of form $\sigma v$, where $Z(\sigma)$ has three connected components, and its identity component has a two-dimensional centre and an adjoint group of type $D_{4}$; the element $v$ of this centralizer has Jordan blocks $J_{1}+J_{1}+J_{3}+J_{3}$.

$U_{0}$ consists of two unipotent classes. One is of type $\begin{gathered}00200 \\ 0\end{gathered}$ (it is the image under $\Phi_{0}$ of the unipotent class in $S_{0}$ ).

The other one is of type $\begin{gathered}20202 \\ 0\end{gathered}$ (it is the image under $\Phi_{0}$ of the nonunipotent class in $S_{0}$ ).

\section{8. $\left(G=G^{*}=F_{4}(k)\right.$.)}

Both $S_{0}$ and $U_{0}$ consist of a single unipotent class, namely that of type 0200. The map $\Phi_{0}$ is the obvious one.

\section{9. $\left(G=G^{*}=G_{2}(k)\right.$.)}

Both $S_{0}$ and $U_{0}$ consist of a single unipotent class, namely the subregular one. The map $\Phi_{0}$ is the obvious one.

1.10. $\left(G=S p_{2 n}(k), G^{*}=S O_{2 n+1}(k), n \geq 2\right.$.)

$S_{0}$ is in natural bijection with the set $\{(A, B) \mid$ ordered, $A \in \diamond, B \in \triangle, n=$ $A+2 B\}$.

To $(A, B)$ corresponds the class of $\sigma v \in S O_{2 n+1}(k)$ where $\sigma$ has centralizer $O_{2 A}(k) \times S O_{4 B+1}(k)$ (connected if and only if $A=0$ ) and $v$ has type

$\left(J_{1}+J_{1}+J_{3}+J_{3}+\cdots+J_{2 a-1}+J_{2 a-1}\right) \times\left(J_{1}+J_{1}+J_{3}+J_{3}+\cdots+J_{2 b-1}+J_{2 b-1}+J_{2 b+1}\right)$ where $a^{2}=A, b^{2}+b=2 B$.

$U_{0}$ is in natural bijection with the set $\{(T, R) \mid$ unordered in $\triangle, n=T+R\}$.

To $(T, R)$ corresponds the unipotent class in $S p_{2 n}(k)$ of type

$$
\left(J_{2}+J_{4}+\cdots J_{2 t}\right)+\left(J_{2}+J_{4}+\cdots+J_{2 r}\right)
$$

where $t^{2}+t=2 T, r^{2}+r=2 R$. 
The map $\Phi_{0}$ is given by

$(A, B)=\left(a^{2},\left(b^{2}+b\right) / 2\right) \mapsto(T, R)=((a+b)(a+b+1) / 2,(a-b)(a-b-1) / 2)$ with $a \geq 0, b \geq 0$.

1.11. $\left(G=\operatorname{Spin}_{2 n+1}(k), G^{*}=P \operatorname{Sp}_{2 n}(k), n \geq 3\right.$.)

$S_{0}$ is in natural bijection with the disjoint union of the sets

$$
\{(A, B) \mid \text { unordered in } \triangle, n=2 \dot{A}+2 B\}
$$

and

$$
\left\{\left(A^{\prime}, B^{\prime}\right) \mid \text { ordered in } \triangle, B^{\prime} \neq 0, n=4 A^{\prime}+B^{\prime}\right\} .
$$

To $(A, B)$ corresponds the class of $\sigma v \in P S p_{2 n}(k)$ where $\sigma$ has a centralizer (connected only if $A \neq B$ ) whose identity component is isogenous to $S p_{4 A}(k) \times S p_{4 B}(k)$ and $v$ has type $\left(J_{2}+J_{2}+J_{4}+J_{4}+\cdots+J_{2 a}+J_{2 a}\right) \times$ $\left(J_{2}+J_{2}+J_{4}+J_{4}+\cdots+J_{2 b}+J_{2 b}\right)$ where $a^{2}+a=2 A, b^{2}+b=2 B$.

To $\left(A^{\prime}, B^{\prime}\right)$ corresponds the class of $\sigma v \in P S p_{2 n}(k)$ where $\sigma$ has a (disconnected) centralizer with identity component doubly covered by $\operatorname{Sp}\left(4 A^{\prime}\right) \times$ $G L\left(B^{\prime}\right) \times S p\left(4 A^{\prime}\right)$ and $v$ has type

$$
\begin{aligned}
\left(J_{2}+\right. & \left.J_{2}+J_{4}+J_{4}+\cdots+J_{2 a}+J_{2 a}\right) \times\left(J_{1}+J_{2}+J_{3}+\cdots+J_{b}\right) \\
& \times\left(J_{2}+J_{2}+J_{4}+J_{4}+\cdots+J_{2 a}+J_{2 a}\right)
\end{aligned}
$$

where $a^{2}+a=2 A^{\prime}, b^{2}+b=2 B^{\prime}$.

$U_{0}$ is in natural bijection with the disjoint union of the sets

$$
\{(T, R) \mid \text { unordered in } \diamond, 2 n+1=T+R\}
$$

and

$\left\{\left(T^{\prime}, R^{\prime}\right) \mid\right.$ unordered, nonconsecutive elements of $\left.\triangle, 2 n+1=T^{\prime}+R^{\prime}\right\}$.

To $(T, R)$ corresponds the unipotent class in $\operatorname{Spin}_{2 n+1}(k)$ of type

$$
\left(J_{1}+J_{3}+\cdots+J_{2 t-1}\right)+\left(J_{1}+J_{3}+\cdots+J_{2 r-1}\right)
$$

where $t^{2}=T, r^{2}=R$.

To $\left(T^{\prime}, R^{\prime}\right)$ corresponds the unipotent class in $\operatorname{Spin}_{2 n+1}(k)$ of type

$$
\left(J_{p_{1}}+J_{p_{2}}+\cdots+J_{p_{m}}\right)+\left(J_{q_{1}}+J_{q_{2}}+\cdots+J_{q_{m^{\prime}}}\right)
$$

where $\left(p_{1}, p_{2}, \ldots, p_{m}\right)$ and $\left(q_{1}, q_{2}, \ldots, q_{m^{\prime}}\right)$ are two arithmetic progressions with initial term 1 or 3 , with ratio 4 and with sum of terms equal to $T^{\prime}, R^{\prime}$ respectively.

The map $\Phi_{0}$ is given by

$$
\begin{aligned}
& (A, B)=\left(\left(a^{2}+a\right) / 2,\left(b^{2}+b\right) / 2\right) \mapsto(T, R)=\left((a+b+1)^{2},(a-b)^{2}\right), \\
& \left(A^{\prime}, B^{\prime}\right)=\left(\left(a^{2}+a\right) / 2,\left(b^{2}+b\right) / 2\right) \\
& \quad \mapsto\left(T^{\prime}, R^{\prime}\right)=((2 a+b+1)(2 a+b+2) / 2,(2 a-b)(2 a-b+1) / 2)
\end{aligned}
$$

with $a \geq 0, b \geq 0$. 
1.12. $\left(G=\operatorname{Spin}_{2 n}(k), G^{*}=P S O_{2 n}(k), n \geq 4\right.$.)

$S_{0}$ is in natural bijection with the disjoint union of the sets

$$
\{(A, B) \mid \text { unordered in } \diamond, A \equiv B \quad(\bmod 2), n=A+B\}
$$

and

$\left\{\left(A^{\prime}, B^{\prime}\right) \mid\right.$ ordered $\left., A^{\prime} \in \diamond, B^{\prime} \in \triangle-\{0\}, A^{\prime} \equiv B^{\prime} \quad(\bmod 2), n=2 A^{\prime}+B^{\prime}\right\}$.

To $(A, B)$ corresponds the class of $\sigma v \in P S O_{2 n}(k)$ where $\sigma$ has a centralizer (connected only for $A \neq B$ ) with identity component isogenous to $\mathrm{SO}_{2 A}(k) \times \mathrm{SO}_{2 B}(k)$ and $v$ has type

$\left(J_{1}+J_{1}+J_{3}+J_{3}+\cdots+J_{2 a-1}+J_{2 a-1}\right) \times\left(J_{1}+J_{1}+J_{3}+J_{3}+\cdots+J_{2 b-1}+J_{2 b-1}\right)$

where $a^{2}=A, b^{2}=B$.

To $\left(A^{\prime}, B^{\prime}\right)$ corresponds the class of $\sigma v \in P S O_{2 n}(k)$ where $\sigma$ has a (disconnected) centralizer with identity component doubly covered by $S O\left(2 A^{\prime}\right) \times$ $G L\left(B^{\prime}\right) \times S O\left(2 A^{\prime}\right)$ and $v$ has type

$$
\begin{aligned}
& \left(J_{1}+J_{1}+J_{3}+J_{3}+\cdots+J_{2 a-1}+J_{2 a-1}\right) \times\left(J_{1}+J_{2}+J_{3}+\cdots+J_{b}\right) \\
& \quad \times\left(J_{1}+J_{1}+J_{3}+J_{3}+\cdots+J_{2 a-1}+J_{2 a-1}\right)
\end{aligned}
$$

where $a^{2}=A^{\prime},\left(b^{2}+b\right) / 2=B^{\prime}$.

$U_{0}$ is in natural bijection with the disjoint union of the sets

$$
\{(T, R) \mid \text { unordered in } \diamond, T \equiv R \equiv 0 \quad(\bmod 2), 2 n=T+R\}
$$

and

$\left\{\left(T^{\prime}, R^{\prime}\right) \mid\right.$ unordered, nonconsecutive elements of $\triangle, T^{\prime} \equiv R^{\prime} \equiv 0(\bmod 2)$,

$$
\left.2 n=T^{\prime}+R^{\prime}\right\}
$$

To $(T, R)$ corresponds the unipotent class in $\operatorname{Spin}_{2 n}(k)$ of type

$$
\left(J_{1}+J_{3}+\cdots+J_{2 t-1}\right)+\left(J_{1}+J_{3}+\cdots+J_{2 r-1}\right)
$$

where $t^{2}=T, r^{2}=R$.

To $\left(T^{\prime}, R^{\prime}\right)$ corresponds the unipotent class in $\operatorname{Spin}_{2 n}(k)$ of type

$$
\left(J_{p_{1}}+J_{p_{2}}+\cdots+J_{p_{m}}\right)+\left(J_{q_{1}}+J_{q_{2}}+\cdots+J_{q_{m^{\prime}}}\right)
$$

where $\left(p_{1}, p_{2}, \ldots, p_{m}\right)$ and $\left(q_{1}, q_{2}, \ldots, q_{m^{\prime}}\right)$ are two arithmetic progressions with initial term 1 or 3 , with ratio 4 and with sum of terms equal to $T^{\prime}, R^{\prime}$ respectively.

The map $\Phi_{0}$ is given by

$$
\begin{gathered}
(A, B)=\left(a^{2}, b^{2}\right) \mapsto(T, R)=\left((a+b)^{2},(a-b)^{2}\right), \\
\left(A^{\prime}, B^{\prime}\right)=\left(\left(a^{2}+a\right) / 2,\left(b^{2}+b\right) / 2\right) \\
\mapsto\left(T^{\prime}, R^{\prime}\right)=((2 a+b)(2 a+b+1) / 2,(2 a-b)(2 a-b+1) / 2)
\end{gathered}
$$

with $a \geq 0, b \geq 0$. 
1.13. The following property of $U_{0}$ can be observed from 1.4-1.12. If $u \in G$ is a unipotent element whose conjugacy class is in $U_{0}$, then

(a) the identity component of the centralizer of $u$ in $G$ is solvable and

(b) $u$ is an even unipotent element, with the single exception of the unipotent of type 010101 in $E_{7}$.

The following property of an element $\sigma v$ in a conjugacy class in $S_{0}$ can be observed from 1.4-1.12.

(c) The centralizer of $\sigma$ in $G^{*}$ is not contained in any Levi subgroup of a proper parabolic subgroup of $G^{*}$ (although its identity component may be contained); moreover, the conjugacy class of $v$ in that centralizer is uniquely determined by $\sigma$.

The following result can be proved by examining each case in 1.4-1.12.

Proposition 1.14. $\Phi_{0}$ defines a bijection $S_{0} \cong U_{0}$.

1.15. We have a natural map from $J_{0}$ to the set of unipotent classes in $G$, obtained by associating to a pair $(C, \mathscr{E})$ the conjugacy class of the unipotent part of some element of $C$. Now the set $J_{0}$ (with the map above) can be explicitly described from the results of [L4, L5]. In particular, we see that the image of this map coincides with $U_{0}$; thus we obtain a surjective map $\Pi: J_{0} \rightarrow U_{0}$.

Now the map $\Psi_{0}: J_{0} \rightarrow S_{0}$ (see 1.2) is defined, but not explicitly computed in [L5]. The following result will describe $\Psi_{0}$ explicitly.

Proposition 1.16. $\Psi_{0}: J_{0} \rightarrow S_{0}$ is the composition of the map $\Pi: J_{0} \rightarrow U_{0}$ with the bijection $U_{0} \rightarrow S_{0}$, inverse to the bijection 1.14 .

(It follows that two cuspidal character sheaves $P_{C, \mathscr{E}}, P_{C^{\prime}, \mathscr{E}^{\prime}}$ are in the same fibre of $\Psi_{0}$ if and only if the unipotent part of any element of $C$ is conjugate to the unipotent part of any element of $C^{\prime}$.)

From [L5] we can find explicitly the cardinals of the fibres of the map $\Psi_{0}$ : $J_{0} \rightarrow S_{0}$ (although the map itself is not explicitly computed in [L5]). From [L4, L5] we can also find explicitly the cardinals of the fibres of the map $\Pi: J_{0} \rightarrow U_{0}$ (this map is explicitly computable).

We find that the fibre of $\Psi_{0}$ at $x \in S_{0}$ has the same number of elements as the fibre of $\Pi$ at $\Phi_{0}(x) \in U_{0}$.

In other words

(a) the two maps $\Pi$ and $\Phi_{0} \Psi_{0}$ from $J_{0}$ to $U_{0}$ have fibres of the same cardinal at any point of $U_{0}$.

We now define a partial order on $U_{0}$ as follows: if $D, D^{\prime} \in U_{0}$, we say that $D \leq D^{\prime}$ if either $D=D^{\prime}$ or $\operatorname{dim} D<\operatorname{dim} D^{\prime}$. The following result is contained in [L9, 10.7(ii)].

(b) For any $j \in J_{0}$ we have $\Pi(j) \leq \Phi_{0} \Psi_{0}(j)$.

We have the following result whose proof is left to the reader.

(c) Let $f, f^{\prime}$ be two maps from a finite set $X_{1}$ to a finite partially ordered set $X_{2}$ such that $f\left(x_{1}\right) \leq f^{\prime}\left(x_{1}\right)$ for all $x_{1} \in X_{1}$ and $\sharp\left(f^{-1}\left(x_{2}\right)\right)=\sharp\left(f^{\prime-1}\left(x_{2}\right)\right)$ for all $x_{2} \in X_{2}$. Then $f=f^{\prime}$.

From (a), (b), (c) it follows formally that $\Pi=\Phi_{0} \Psi_{0}$ as maps from $J_{0}$ to $U_{0}$. This, together with 1.14 , clearly implies the proposition. 
1.17. According to [L5, III 11.5], to any character sheaf $A$ on $G$ corresponds a homomorphism $\theta_{A}: \mathscr{Z} \rightarrow \bar{Q}_{l}^{*}$. Assume that $A=P_{C, \mathscr{E}}$ is a cuspidal character sheaf. Then $\theta_{A}$ can be defined as follows. Let $f, f^{\prime}: G \times C \rightarrow C$ be defined by $f(g, c)=g c g^{-1}$ and $f^{\prime}(g, c)=c$. Let $h: C \rightarrow G \times C$ be defined by $h(c)=(1, c)$. We have $f h=f^{\prime} h=\operatorname{Id}_{C}$. There is a unique isomorphism of local systems $l: f^{* *} \mathscr{E} \cong f^{*} \mathscr{E}$ over $G \times C$ such that the induced isomorphism between $h^{*} f^{\prime *} \mathscr{E}=\mathscr{E}$ and $h^{*} f^{*} \mathscr{E}=\mathscr{E}$ is the identity $\mathscr{E} \rightarrow \mathscr{E}$.

Now $l$ defines for any $(g, c) \in G \times C$, an isomorphism of stalks $l_{g, c}: \mathscr{E}_{c} \cong$ $\mathscr{E}_{\mathrm{gcg}^{-1}}$. The following identity follows easily from definitions:

(a) $l_{g_{1} g_{2}, c}=l_{g_{1}, g_{2} c g_{2}^{-1} l_{g_{2}, c}}$, for any $g_{1}, g_{2} \in G$ and any $c \in C$.

In particular, if $g_{1}, g_{2}$ belong to $Z_{G}(c)$, the centralizer of $c$, then (a) shows that $g_{1} \mapsto l_{g_{1}, c}$ is a representation of the group of components of $Z_{G}(c)$ on the stalk $\mathscr{E}_{c}$. This representation is in fact irreducible, since the local system $\mathscr{E}$ is irreducible.

It follows that, if $g \in \mathscr{Z}$, then $l_{g, c}: \mathscr{E}_{c} \cong \mathscr{E}_{c}$ is multiplication by a scalar $\chi_{A}(g) \in \bar{Q}_{l}^{*}$; this scalar is independent of $c$ and $g \mapsto \chi_{A}(g)$ is a homomorphism $\mathscr{Z} \rightarrow \bar{Q}_{l}^{*}$. This defines $\theta_{A}$. We shall also write $\theta_{C, \mathscr{E}}$ instead of $\theta_{A}$.

Thus we have a map $(C, \mathscr{E}) \mapsto \theta_{C, \mathscr{E}}$ from $J_{0}$ to $\operatorname{Hom}\left(\mathscr{Z}, \bar{Q}_{l}^{*}\right)$. Let $J_{0, \theta}$ be the inverse image under this map of an element $\theta$. We have a partition

(b) $J_{0}=\sqcup_{\theta} J_{0, \theta}$.

1.18. For any $z \in \mathscr{Z}$ we denote by $t_{z}: G \rightarrow G$ the map $g \rightarrow z g$. If $(C, \mathscr{E}) \in$ $J_{0}$, then $\left(C^{\prime}, \mathscr{E}^{\prime}\right):=\left(z^{-1} C, t_{z}^{*} \mathscr{E}\right)$ is again in $J_{0}$ and it is easy to see that $\theta_{C^{\prime}, \mathscr{E}^{\prime}}=\theta_{C, \mathscr{E}}$.

Thus, $(C, \mathscr{E}) \mapsto\left(z^{-1} C, t_{z}^{*} \mathscr{E}\right)$ defines an action of $\mathscr{Z}$ on $J_{0}$ which leaves stable each of the subsets $J_{0, \theta}$ (see 1.15(a)).

Consider now the fibre $\Pi^{-1}(D)$ of the map $\Pi: J_{0} \rightarrow U_{0}$ at an element $D$ of $U_{0}$. For each $\theta \in \hat{\mathcal{Z}}$, let $\Pi^{-1}(D)_{\theta}=\Pi^{-1}(D) \cap J_{0, \theta}$.

It is clear that the $\mathscr{Z}$-action on $J_{0}$ leaves stable each of the subsets $\Pi^{-1}(D)$, hence also each of the subsets $\Pi^{-1}(D)_{\theta}$.

In 1.19-1.25 we give some information on the subsets $\Pi^{-1}(D)_{\theta}$ and the $\mathscr{Z}$-action on them, which can be extracted from [L5].

1.19. $\left(G=S L_{n}(k), n \geq 2\right.$.) The set $J_{0, \theta}$ is nonempty precisely when $\chi$ : $\mathscr{Z} \rightarrow \bar{Q}_{l}^{*}$ is injective; in that case, $\mathscr{Z} \cong Z / n Z$ acts simply transitively on $J_{0, \theta}$. Hence the set $J_{0}$ has $n \phi(n)$ elements, where $\phi$ is Euler's function.

1.20. $\left(G=E_{8}(k), F_{4}(k)\right.$ or $G_{2}(k)$.) The set $J_{0}$ has respectively $13,7,4$ elements.

1.21. $\left(G=E_{7}(k)^{\mathrm{sc}}\right.$.) If $D$ is the unipotent class 0100101 , then $\Pi^{-1}(D)=$ $\Pi^{-1}(D)_{\theta}$ where $\theta$ is the trivial character of $\mathscr{Z} \cong Z / 2 Z$; this set has cardinal 2 and $\mathscr{Z}$ acts trivially on it.

If $D$ is the unipotent class 200200 , then $\Pi^{-1}(D)=\Pi^{-1}(D)_{\theta}$ where $\theta$ is 
the nontrivial character of $\mathscr{Z}$; this set has cardinal 6 and $\mathscr{Z}$ acts freely (with three orbits) on it.

1.22. $\left(G=E_{6}(k)^{\mathrm{sc}}\right.$.) If $D$ is the unipotent class 00200 , then $\Pi^{-1}(D)=$ $\Pi^{-1}(D)_{\theta}$ where $\theta$ is the trivial character of $\mathscr{Z} \cong Z / 3 Z$; this set has cardinal 2 and $\mathscr{Z}$ acts trivially on it.

If $D$ is the unipotent class 20202 , then $\Pi^{-1}(D)=\Pi^{-1}(D)_{\theta} \sqcup \Pi^{-1}(D)_{\theta^{\prime}}$ where $\theta$ and $\theta^{\prime}$ are the two nontrivial character of $\mathscr{Z}$; the two sets $\Pi^{-1}(D)_{\theta}$, $\Pi^{-1}(D)_{\theta^{\prime}}$ have six elements each, and $\mathscr{Z}$ acts freely (with two orbits) on each. 1.23. $\left(G=S p_{2 n}(k), n \geq 2\right.$.) We have $J_{0}=J_{0, \theta}$ where $\theta$ is trivial if $n$ is even, and $\theta$ is nontrivial if $n$ is odd.

The action of $\mathscr{Z} \cong Z / 2 Z$ on $\Pi^{-1}(D)$ is transitive; it is free if $D$ corresponds to $(T, R)$ with $T \neq R$ and is trivial if $D$ corresponds to $(T, R)$ with $T=R$ (see 1.10).

1.24. $\left(G=\operatorname{Spin}_{2 n+1}(k), n \geq 3\right.$.) If $D$ corresponds to $(T, R)$ with $T>$ $0, R>0$, then $\Pi^{-1}(D)=\Pi^{-1}(D)_{\theta}$ where $\theta$ is the trivial character of $\mathscr{Z} \cong$ $Z / 2 Z$; this set has exactly one element and $\mathscr{Z}$ acts trivially on it.

If $D$ corresponds to $(T, R)$ with $T=0$ or $R=0$, then $\Pi^{-1}(D)=\Pi^{-1}(D)_{\theta}$ where $\theta$ is the nontrivial character of $\mathscr{Z}$ and $\mathscr{Z}$ acts simply transitively on $\Pi^{-1}(D)$.

If $D$ corresponds to $\left(T^{\prime}, R^{\prime}\right)$, then $\Pi^{-1}(D)=\Pi^{-1}(D)_{\theta} \sqcup \Pi^{-1}(D)_{\theta^{\prime}}$ where $\theta, \theta^{\prime}$ are the two characters of $\mathscr{Z}$; the two sets $\Pi^{-1}(D)_{\theta}, \Pi^{-1}(D)_{\theta^{\prime}}$ have two elements each, and $\mathscr{Z}$ acts simply transitively on both.

1.25. ( $G=\operatorname{Spin}_{2 n}(k), n \geq 4$.) Let $\epsilon$ be the nontrivial element in the kernel of the natural homomorphism $G \rightarrow S O_{2 n}(k)$ (for $n>4$ ).

If $D$ corresponds to $(T, R)$ with $T=R$, then $\Pi^{-1}(D)=\Pi^{-1}(D)_{\theta}$ where $\theta$ is the trivial character of $\mathscr{Z}$; this set has one element and $\mathscr{Z}$ acts trivially on it.

If $D$ corresponds to $(T, R)$ with $T \neq R$ and $T>0, R>0$, then $n$ is even, $n>4$ and $\Pi^{-1}(D)=\Pi^{-1}(D)_{\theta}$ where $\theta$ is determined by the condition that $\theta(\epsilon)=1$ and by the condition that $\theta$ is trivial precisely when $n$ is divisible by 4; the set $\Pi^{-1}(D)$ has cardinal 2 and the action of $\mathscr{Z}$ on it is transitive, with $\epsilon$ acting trivially.

If $D$ corresponds to $(T, R)$ with $T=0$ or $R=0$ and $n \equiv 2(\bmod 4)$ then $n>4$ and $\Pi^{-1}(D)=\Pi^{-1}(D)_{\theta}$ where $\theta$ is the nontrivial character of $\mathscr{Z}$ which takes the value 1 on $\epsilon$; the action of $\mathscr{Z}$ on $\Pi^{-1}(D)$ is simply transitive.

If $D$ corresponds to $(T, R)$ with $T=0$ or $R=0$ and $n \equiv 0(\bmod 4)$ then $n>4$ and $\Pi^{-1}(D)=\Pi^{-1}(D)_{\theta} \sqcup \Pi^{-1}(D)_{\theta^{\prime}} \sqcup \Pi^{-1}(D)_{\theta^{\prime \prime}}$ where $\theta$ is the trivial character of $\mathscr{Z}$ and $\theta^{\prime}, \theta^{\prime \prime}$ are the two characters of $\mathscr{Z}$ which are nontrivial on $\epsilon$; the three sets $\Pi^{-1}(D)_{\theta}, \Pi^{-1}(D)_{\theta^{\prime}}, \Pi^{-1}(D)_{\theta^{\prime \prime}}$ have four elements each and $\mathscr{Z}$ acts simply transitively on each.

If $D$ corresponds to $\left(T^{\prime}, R^{\prime}\right)$ with $T^{\prime}=R^{\prime}$, then $n$ is even, $n>4$ and $\Pi^{-1}(D)=\Pi^{-1}(D)_{\theta^{\prime}} \sqcup \Pi^{-1}(D)_{\theta^{\prime \prime}}$ where $\theta^{\prime}, \theta^{\prime \prime}$ are the two characters of $\mathscr{Z}$ 
which are nontrivial on $\epsilon$; the two sets $\Pi^{-1}(D)_{\theta^{\prime}}, \Pi^{-1}(D)_{\theta^{\prime \prime}}$ have two elements each, and $\mathscr{Z}$ acts transitively on both in such a way that the kernel of $\theta^{\prime}$ acts trivially on $\Pi^{-1}(D)_{\theta^{\prime}}$ and the kernel of $\theta^{\prime}$ acts trivially on $\Pi^{-1}(D)_{\theta^{\prime \prime}}$.

If $D$ corresponds to $\left(T^{\prime}, R^{\prime}\right)$ with $T^{\prime} \neq R^{\prime}$, then $n>4$ and $\Pi^{-1}(D)=$ $\Pi^{-1}(D)_{\theta^{\prime}} \sqcup \Pi^{-1}(D)_{\theta^{\prime \prime}}$ where $\theta^{\prime}, \theta^{\prime \prime}$ are the two characters of $\mathscr{Z}$ which are nontrivial on $\epsilon$; the two sets $\Pi^{-1}(D)_{\theta^{\prime}}, \Pi^{-1}(D)_{\theta^{\prime \prime}}$ have four elements each, and $\mathscr{Z}$ acts simply transitively on both.

1.26. From $1.19-1.25$ we see that in every case, the isotropy group of the $\mathscr{Z}$ action on $\Pi^{-1}(D)_{\theta}$ (assumed to be nonempty) is contained in the kernel of $\theta: \mathscr{Z} \rightarrow \bar{Q}_{l}$. Moreover, if $G$ is a classical group, $\mathscr{Z}$ acts transitively on $\Pi^{-1}(D)_{\theta}$, if that set is nonempty.

\section{2. $F_{q}$-RATIONAL STRUCTURES}

2.1. From now on, we assume that $G$ is simply connected, almost simple. Let $F_{q}$ be the finite subfield of $k$ with $q$ elements. We assume that we are given an $F_{q}$-rational structure on $G$ with Frobenius map $F: G \rightarrow G$. Then $G^{*}$ inherits from $G$ an $F_{q}$-rational structure with Frobenius map $F: G^{*} \rightarrow G^{*}$. We denote by $G^{F}$ the fixed point set of $F: G \rightarrow G$.

Recall that the subset $\mathbf{B}_{2}$ of $\mathscr{A}$ has been defined in 0.4; it is naturally in 1-1 correspondence with the set $J_{0}^{F}$ of fixed points of the Frobenius map, which acts naturally on $J_{0}$.

Proposition 2.2. The subset $\mathbf{B}_{2}$ is a basis of $\mathscr{A}^{0}$ (see 0.7).

Let $\mathscr{A}_{1}\left(\right.$ resp. $\left.\mathscr{A}_{0}\right)$ be the subspace of $\mathscr{A}$ spanned by $\mathbf{B}_{1}-\mathbf{B}_{2}\left(\right.$ resp. $\left.\mathbf{B}_{2}\right)$. (See $0.3,0.4$.) Let $\mathscr{A}^{1}$ be the subspace of $\mathscr{A}$ spanned by all $R_{L}^{G}(f)$ (see 0.4 ) for various proper $L$ defined over $F_{q}$ and various class functions $f$ of $L\left(F_{q}\right)$; then $\mathscr{A}^{0}$ consists of the class functions orthogonal to $\mathscr{A}^{1}$.

From the results of [L5] we have that $\mathscr{A}_{0}$ consists of all class functions orthogonal to $\mathscr{A}_{1}$. From [L8, 9.5] we have that $\mathscr{A}_{1} \subset \mathscr{A}^{1}$. Taking orthogonals, we deduce $\mathscr{A}^{0} \subset \mathscr{A}_{0}$.

Next we note that, by $[L 8,9.5]$ and the transitivity of twisted induction, $\mathscr{A}^{1}$ is spanned by functions of form $R_{L}^{G}(f)$ with $L$ proper and $f: L\left(F_{q}\right) \rightarrow \bar{Q}_{l}$ in B $_{2}$ (relative to $L$ ); such $R_{L}^{G}(f)$ is orthogonal to any function in $\mathscr{A}_{0}$, by [L8, 9.2] and the orthogonality relations for characteristic functions of characted sheaves [L5]. Thus, $\mathscr{A}^{1}$ is orthogonal to $\mathscr{A}_{0}$, hence $\mathscr{A}_{0} \subset \mathscr{A}^{0}$. Thus we have $\mathscr{A}^{0}=\mathscr{A}_{0}$. (Compare [L8, 1.14(b)].) Since $\mathbf{B}_{2}$ is a basis of $\mathscr{A}_{0}$, the proposition follows.

2.3. We have a canonical surjective map $\Theta^{\prime}$ from $\mathbf{A}_{0}$ (see 0.1 ) to the set of special conjugacy classes in $G^{*}$ which are defined over $F_{q}$ (see [L3, L7]). If $\beta \in \mathbf{A}_{2}$ (see 0.7) then, by definition, $\beta$ is a linear combination of irreducible characters $b$ in a fixed fibre of $\Theta^{\prime}$; hence we may define $\Theta(\beta)$ to be $\Theta^{\prime}(b)$ 
for any $b$ as above. We thus have a surjective map $\Theta$ from $\mathbf{A}_{2}$ to the set of special conjugacy classes in $G^{*}$ which are defined over $F_{q}$. From the explicit knowledge of $\mathbf{A}_{2}$ we see that $\Theta\left(\mathbf{A}_{2}\right) \subset S_{0}$ where $S_{0}$ is as in 1.2. Composition with the bijection $\Phi_{0}: S_{0} \rightarrow U_{0}$ (see (see 1.3,1.14) gives a map $\Phi_{0} \Theta: \mathbf{A}_{2} \rightarrow U_{0}$.

For any $D \in U_{0}$, we denote by $\mathscr{A}^{0}(D)$ the subspace of $\mathscr{A}^{0}$ spanned by $\mathbf{A}_{2}(D)=\left\{\beta \in \mathbf{A}_{2} \mid \Phi_{0} \Theta(\beta)=D\right\} ;$ we denote by $\mathscr{A}^{0}[D]$ the subspace of $\mathscr{A}^{0}$ spanned by the set $\mathbf{B}_{2}(D)$ consisting of all $b \in \mathbf{B}_{2}$ such that the corresponding element $(C, \mathscr{E}) \in J_{0}$ satisfies $\Pi(C, \mathscr{E})=D$ (see 1.15).

Proposition 2.4. For any $D \in U_{0}$, we have $\mathscr{A}^{0}(D)=\mathscr{A}^{0}[D]$.

The number of elements in the two sets $\mathbf{A}_{2}(D)$ and $\mathbf{B}_{2}(D)$ is explicitly computable (for $\mathbf{B}_{2}(D)$ this is essentially contained in 1.19-1.25); this number is the same in both cases. Hence $\operatorname{dim} \mathscr{A}^{0}(D)=\operatorname{dim} \mathscr{A}^{0}[D]$.

From the results on unipotent support of irreducible representations given in $[L 9,11.2]$, any $\beta \in \mathbf{A}_{2}(D)$ can have nonzero values only at elements with unipotent part contained in a unipotent class $D_{1}$ of $G$ such that $D_{1}=D$ or $\operatorname{dim} D_{1}<\operatorname{dim} D$. Since $\beta$ is contained in the space spanned by $\mathbf{B}_{2}$ (see 2.2 ), it must be contained in the subspace $\sum \mathscr{A}^{0}\left[D_{1}\right]$ where $D_{1}$ runs over the elements of $U_{0}$ which are $\leq D$ for the partial order in the proof of 1.16. Thus, for any $D \in U_{0}$, we have $\mathscr{A}^{0}(D) \subset \sum_{D_{1} \leq D} \mathscr{A}^{0}\left[D_{1}\right]$; hence $\sum_{D_{1} \leq D} \mathscr{A}^{0}\left(D_{1}\right) \subset$ $\sum_{D_{1} \leq D} \mathscr{A}^{0}\left[D_{1}\right]$. The last inclusion is between spaces of equal dimension (as remarked earlier); hence the inclusion must be an equality $\sum_{D_{1} \leq D} \mathscr{A}^{0}\left(D_{1}\right)=$ $\sum_{D_{1} \leq D} \mathscr{A}^{0}\left[D_{1}\right]$. Since in both sums the summands are orthogonal to each other, the last equality implies by induction on $\operatorname{dim} D$ that $\mathscr{A}^{0}(D)=\mathscr{A}^{0}[D]$. The proposition is proved. (Compare with the proof of 1.16.)

\section{THE $\mathscr{Z}^{F} \times \mathscr{Z}_{F}$-ACTION}

3.1. For any $z^{\prime} \in \mathscr{Z}_{F}$ we define a group automorphism as follows: we represent $z^{\prime}$ by $\dot{z} \in \mathscr{Z}$ and we write $\dot{z}=\xi^{-1} F(\xi)$ with $\xi \in G$; we set $\gamma_{z^{\prime}}(g)=\xi g \xi^{-1}$. (This is independent of the choices.) We define a $\bar{Q}_{l}$-linear map $r_{z^{\prime}}: \mathscr{A} \rightarrow \mathscr{A}$ by $\left(r_{z^{\prime}} f\right)(g)=f\left(\gamma_{z^{\prime}}(g)\right)$ for all $f \in \mathscr{A}$ and $g \in G^{F}$.

It is clear that $\left(z, z^{\prime}\right) \mapsto t_{z} r_{z^{\prime}}: \mathscr{A} \rightarrow \mathscr{A}$ is a linear representation of the finite group $\mathscr{Z}^{F} \times \mathscr{Z}_{F}$ on the $\bar{Q}_{l}$-vector space $\mathscr{A}$.

Lemma 3.2. Let $(C, \mathscr{E}) \in J_{0}^{F}$ and let $\pi$ and $\chi_{C, \mathscr{E}, \pi} \in \mathscr{A}$ be as in 0.2.

For any $z^{\prime} \in \mathscr{Z}$, we have $r_{z^{\prime}} \chi_{C, \mathscr{E}, \pi}=\theta_{C, \mathscr{E}}(\dot{z}) \chi_{C, \mathscr{E}, \pi}$ where $\dot{z}$ is any representative of $z^{\prime}$ in $\mathscr{Z}$.

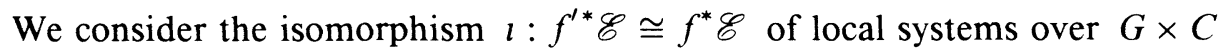
(see 1.17).

We define an isomorphism $\imath^{\prime}: f^{\prime *} \mathscr{E} \cong f^{*} \mathscr{E}$ of local systems over $G \times C$ as 
the composition of isomorphisms

$$
f^{*} \mathscr{E} \cong f^{\prime *} F^{*} \mathscr{E} \cong F^{*} f^{*} \mathscr{E} \cong F^{*} f^{*} \mathscr{E} \cong f^{*} F^{*} \mathscr{E} \cong f^{*} \mathscr{E}
$$

(the first one is obtained by applying $f^{\prime *}$ to $\pi$; the second and fourth are the obvious ones; the third is obtained by applying $F^{*}$ to $l$; the fifth one is obtained by applying $f^{*}$ to $\pi^{-1}$ ).

It is clear that $l^{\prime}$ satisfies the defining property of $l$ (see 1.17); hence, by uniqueness, it coincides with $l$.

This implies, by passing to stalks that, for any $(g, c) \in G \times C$,

(a) the isomorphism $l_{g, c}: \mathscr{E}_{c} \cong \mathscr{E}_{g c g^{-1}}$ (see 1.17) is equal to the composition of isomorphisms

$$
\mathscr{E}_{c} \cong \mathscr{E}_{F c} \cong \mathscr{E}_{F\left(g c g^{-1}\right)} \cong \mathscr{E}_{g c g^{-1}}
$$

(the first one is $\pi_{c}$ (see $0.3(\mathrm{a})$ ); the second one is $l_{F g}, F c$; the third one is $\pi_{g c g^{-1}}^{-1}$.

We apply (a) to $c \in C^{F}$ and to $g \in G$ such that $g^{-1} F(g)=z_{1}$ where $z_{1} c=c z_{1}$; note that, by $1.17(\mathrm{a})$, we have $l_{F g, F c}=l_{g z_{1}, c}=l_{g, c z_{1}, c}$. Hence from (a) we see that

(b) $\pi_{g c g^{-1}}=l_{g, c} l_{z_{1}, c} \pi_{c} l_{g, c}^{-1}$.

Taking traces we deduce that

(c) $\operatorname{tr}\left(\pi_{g c g^{-1}}, \mathscr{E}_{g c g^{-1}}\right)=\operatorname{tr}\left(l_{z_{1}, c} \pi_{c}, \mathscr{E}_{c}\right)$.

Assume now that $z_{1}=\dot{z}$ where $\dot{z} \in \mathscr{Z}$ represents $z^{\prime}$. Then from (c) we deduce

$$
r_{z^{\prime}} \chi_{C, \mathscr{E}, \phi}(c)=\theta_{C, \mathscr{E}}(\dot{z})^{-1} \chi_{C, \mathscr{E}, \pi}(c)
$$

for any $c \in C^{F}$. The same equality holds trivially for $c \in G^{F}-C^{F}$ (both sides of it are zero). The lemma is proved.

3.3. Let $\mathscr{Z}^{F}$ be the fixed point set of $F: \mathscr{Z} \rightarrow \mathscr{Z}$. It follows immediately from the definitions that, for any $z \in \mathscr{Z}^{F}$, the automorphism $t_{z}: \mathscr{A} \rightarrow \mathscr{A}$ (see 0.6) maps any element of $b \in \mathbf{B}_{2}$ to some element $b^{\prime} \in \mathbf{B}_{2}$ times a root of 1. Moreover, it is clear that, if $b \in \mathbf{B}_{2}(D)$, then $b^{\prime} \in \mathbf{B}_{2}(D)$.

We have the following result.

Lemma 3.4. Let $b \in \mathbf{B}_{2}$ and $z \in \mathscr{Z}^{F}$ be such that $t_{z} b$ is a multiple of $b$ (see 3.3). Let $(C, \mathscr{E})$ be the element of $J_{0}$ corresponding to $b$. Let $\bar{G}$ the quotient of $G$ by the subgroup of $\mathscr{Z}$ generated by $z$ and let $\operatorname{pr}: G \rightarrow \bar{G}$ be the natural map.

(a) There exists a cuspidal pair $(\bar{C}, \overline{\mathscr{E}})$ for $\bar{G}$ such that $F(\bar{C})=\bar{C}, \overline{\mathscr{E}} \cong$ $F^{*} \overline{\mathscr{E}}, \operatorname{pr}^{-1}(\bar{C})=C$ and $\operatorname{pr}^{*}(\overline{\mathscr{E}}) \cong \mathscr{E}$.

(b) We have $t_{z} b=b$.

(a) can be extracted from the results of [L5]; (b) follows clearly from (a).

3.5. From 3.3 and $3.4(\mathrm{a})$ it follows that, by changing if necessary the normalization of the elements of $\mathbf{B}_{2}$, we may assume that $t_{z}\left(\mathbf{B}_{2}(D)\right)=\mathbf{B}_{2}(D)$ for any $z \in \mathscr{Z}^{F}$ and $D \in U_{0}$. 
3.6. The action of $t_{z}, r_{z^{\prime}}$ on $\mathbf{A}_{0}$ is explicitly known; from this we see that this action has the following properties.

(a) We have $t_{z}\left(\mathbf{A}_{2}(D)\right)=\mathbf{A}_{2}(D)$ for any $z \in \mathscr{Z}^{F}$ and $D \in U_{0}$.

(b) For any $\beta \in \mathbf{A}_{2}(D)$ and any $z^{\prime} \in \mathscr{Z}_{F}$, we have $r_{z^{\prime}} \beta=\mu \beta$ for some $\mu$, a root of 1 .

3.7. We can now define $\mathbf{B}_{3}$ and $\mathbf{A}_{3}$ as in 0.6, 0.7. Let $\mathbf{B}_{3}(D)=\mathbf{B}_{3} \cap \mathscr{A}^{0}(D)$ and $\mathbf{A}_{3}(D)=\mathbf{A}_{3} \cap \mathscr{A}^{0}(D)$. It is clear that $\mathbf{B}_{3}(D), \mathbf{A}_{3}(D)$ are two bases of $\mathscr{A}^{0}(D)$.

From the definitions and from 3.5, 3.6, we see that, for any $D \in U_{0}$,

(a) each element of $\mathbf{B}_{3}(D)$ and each element of $\mathbf{A}_{3}(D)$ is an eigenvector for the action $\left(z, z^{\prime}\right) \mapsto t_{z} r_{z^{\prime}}$ of $\mathscr{Z}^{F} \times \mathscr{Z}_{F}$ on $\mathscr{A}^{0}(D)$.

3.8. We now prove Theorem 0.8 assuming that $G$ has rank $\geq 9$. Then, from $1.19,1.23-1.25$, it follows easily that all eigenspaces for the $\mathscr{Z}^{F} \times \mathscr{Z}_{F}$-action on $\mathscr{A}^{0}(D)$ are one dimensional, except in the following case:

(a) $G=\operatorname{Spin}_{2 n}(k)$ (nonsplit over $F_{q}$ ), $n$ even and $D$ corresponds as in 1.12 to $(T, R)$ with $T \neq R, T>0, R>0$.

Assume first that we are not in case (a). Using 3.7(a) we deduce that the elements of $\mathbf{B}_{3}(D)$ are the same up to nonzero scalar factors as the elements of $\mathbf{A}_{3}(D)$.

Let $b \in \mathbf{B}_{3}(D)$ and $\beta \in \mathbf{A}_{3}(D)$ be corresponding elements (i.e., elements in the same eigenspace). Then $b=\mu \beta$ where $\mu \in \bar{Q}_{l}^{*}$.

If $G \cong S L_{n}(k)$, we may assume that $n$ divides $q-\delta$, where $\delta=1$ if $G$ is split over $F_{q}$ and $\delta=-1$ if $G$ is nonsplit over $F_{q}$ (otherwise, $J_{0}^{F}$ is empty and there is nothing to prove). Then $\beta$ is a linear combination of $n$ distinct irreducible characters with coefficients $n$th roots of 1 . On the other hand, by a variant of [L9, 7.6], $b$ can be normalized so that it is a linear combination of irreducible characters with coefficients in the group generated by the roots of 1 . It follows that $\mu$ must belong to this last group. Since the self inner product of $b$ is $n$ and the self inner product of $\beta$ is $n$ it follows that $\mu \bar{\mu}=1$ where $-: \bar{Q}_{l} \rightarrow \bar{Q}_{l}$ is a field involution which takes each root of 1 to its inverse. It follows that $\mu$ is a root of 1 , as required.

If $G$ is not $S L_{n}(k)$, then $\beta$ is a linear combination of $2^{s}$ distinct irreducible characters with coefficients of the form $2^{-t} \omega$ where $\omega^{4}=1$ and $t$ is an integer $\geq 0$.

On the other hand, by a variant of [L9, 7.6], $b$ can be can be normalized so that $2^{t} b$ is a linear combination of irreducible characters with coefficients in the group generated by the 4 th roots of 1 . It follows that $\mu$ must belong to this last group. Since the self inner product of $b$ is $2^{s-2 t}$ and the self inner product of $\beta$ is $2^{s-2 t}$ it follows that $\mu \bar{\mu}=1$ and hence $\mu^{4}=1$.

Assume next that we are in case (a). Then $\operatorname{dim} \mathscr{A}^{0}(D)=2$. Let $b, b^{\prime}$ be the two elements of $\mathbf{B}_{2}(D)=\mathbf{B}_{3}(D)$ and let $\beta, \beta^{\prime}$ be the two elements of $\mathbf{A}_{2}(D)=\mathbf{A}_{3}(D)$. Let $P$ be the set of all $f \in \mathscr{A}^{0}(D)$ which are integral linear combinations of irreducible characters. 
We note that each of $\beta, \beta^{\prime}$ is a linear combinations of $2^{2 t}$ distinct irreducible characters with coefficients of the form $\pm 2^{-t}$ and $t$ is an integer $\geq 1$. Hence $2^{t} \beta$ and $2^{t} \beta^{\prime}$ belong to $P$ and have self inner product $2^{2 t}$. One verifies that, conversely, any element of $P$ with self inner product $2^{2 t}$ is either $\pm \beta$ or $\pm \beta^{\prime}$. By a variant of $[\mathrm{L} 9,7.6], b, b^{\prime}$ can be normalized so that $2^{t} b$ and $2^{t} b^{\prime}$ are integral linear combinations of irreducible characters. Thus, they belong to $P$. It is clear that $2^{t} b$ and $2^{t} b^{\prime}$ have self inner product $2^{2 t}$. It follows that we can arrange notation so that $2^{t} \beta= \pm 2^{t} b$ and $2^{t} \beta^{\prime}= \pm 2^{t} b^{\prime}$; hence $\beta= \pm b$ and $\beta^{\prime}= \pm b^{\prime}$.

\section{REFERENCES}

[A] T. Asai, The unipotent class functions on the symplectic groups and the odd orthogonal groups over finite fields, Comm. Algebra 12 (1984), 617-645.

[BS] W. M. Beynon and N. Spaltenstein, Green functions on finite Chevalley groups of type $E_{n}$ $(n=6,7,8)$, J. Algebra 88 (1984), 584-614.

[DL1] P. Deligne and G. Lusztig, Representations of reductive groups over a finite field, Ann. of Math. (2) 103 (1976), 103-161.

[K1] N. Kawanaka, Generalized Gelfand-Graev representations of exceptional simple algebraic groups over a finite field, Invent. Math. 84 (1986), 575-616.

[K2] _ Shintani lifting and Gelfand-Graev representations, Proc. Sympos. Pure Math., vol. 47, Amer. Math. Soc., Providence, RI, 1987, pp. 147-163.

[L1] G. Lusztig, On the finiteness of the number of unipotent classes, Invent. Math 34 (1976), 201-213.

[L2] _ A class of irreducible representations of a Weyl group, Nederl. Akad. Wetensch. Proc. Ser. A 82 (1979), 323-335.

[L3] _ Characters of reductive groups over a finite field, Ann. of Math. Stud., vol. 107, Princeton Univ. Press, Princeton, NJ, 1984.

[L4] Intersection cohomology complexes on a reductive group, Invent. Math. 75 (1984), 205-272.

[L5] _ Character sheaves, Adv. in Math. 56 (1985), 193-237;II, 57 (1985), 226-265; III, 57 (1985), 266-315; IV, 59 (1986), 1-63; V, 61 (1986), 103-155.

[L6] - On the character values of finite Chevalley groups at unipotent elements, J. Algebra 104 (1986), 146-194.

[L7] _ On representations of reductive groups with disconnected center, Astérisque $\mathbf{1 6 8}$ (1988), 157-166.

[L8] _ Green functions and character sheaves, Ann. of Math. (2) 131 (1990), 355-408.

[L9] _ A unipotent support for irreducible representations, Adv. in Math (to appear).

[S1] T. Shoji, On the Green polynomials of classical groups, Invent. Math. 74 (1983), 239-264.

[S2] - Some generalization of Asai's result for classical groups, Algebraic Groups and Related Topics, Adv. Stud. Pure Math., vol. 6, Kinokuniya and North Holland, Tokyo and Amsterdam, 1985.

Department of Mathematics, Massachusetts institute of Technology, Cambridge, MASSACHUSETTS 02139 